\title{
Enzyme immunoassay for anti-treponemal IgG: Screening or confirmatory test?
}

\author{
H Young, A Moyes, A McMillan, J Patterson
}

\begin{abstract}
Aims: To review the performance of the Venereal Diseases Research Laboratory (VDRL) test and the Treponema pallidum haemagglutination assay (TPHA) as a combined screen for syphilis to provide a baseline for assessing screening by anti-treponemal IGG EIA.

Methods: Between 1980 and 1987 all serum samples were screened by both VDRL and TPHA tests. The FTA-ABS test was also used in suspected early primary syphilis, or when one of the other tests was positive. A positive result in a screening test was confirmed by quantitative testing. From 1988 all specimens were screened with an enzyme immunoassay (Captia Syph G) as a single screening test.
\end{abstract}

Results: Of the 44 primary, 47 secondary, and 38 early latent cases of syphilis, the VDRL and TPHA detected $32(73 \%)$ and $31(71 \%)$ of the primary cases; the combination detected 37 (84\%). All 85 cases of cases of secondary and early latent infection were reactive in the TPHA test, whereas the VDRL was reactive in only $68(80 \%)$. EIA had a reported sensitivity of $82 \%$ for primary infection.

Conclusions: EIA can be used as a single screening test for detecting early syphilis because its results are comparable with those of the combined VDRL and TPHA tests. The conventional VDRL test should not be used as a single screening test.

The addition of the enzyme immunoassay (EIA) to the range of serological tests for treponemal infection offers laboratories yet greater choice in the selection of individual tests or combinations of tests for syphilis screening and diagnosis. ${ }^{1}$ A possible limitation of screening with an anti-treponemal IgG EIA is the failure to detect cases of primary syphilis. The natural humoral response to treponemal infection means that there is a period of two to three weeks when the IgG response may be absent or inadequate for serological detection. ${ }^{2}$ Although more data are required, this limitation may be more of a theoretical rather than a practical issue. Nevertheless, it is important to consider when deciding whether EIAs that detect antitreponemal IgG should be used as screening ${ }^{3}$ or as confirmatory tests. ${ }^{4}$

The potential for automation makes EIA a particularly attractive screening test as we reported in our evaluation ${ }^{3}$ of Captia Syph G (Mercia Diagnostics UK). The overall screening performance of this new anti-treponemal IgG EIA (sensitivity $98.4 \%$ and specificity $99.3 \%$ ) was comparable with that provided by the widely used screening combination of the Venereal Diseases Research Laboratory (VDRL) test and the Treponema pallidum haemagglutination assay (TPHA). Unfortunately, there were too few cases of primary syphilis in our patient population to assess the efficiency of Captia Syph G in detecting primary infection. Recently, Lefèvre and colleagues evaluated the Captia Syph G in 96 patients with untreated syphilis at various stages, including 17 patients with primary stage infection as well as 63 patients with treated infection. ${ }^{4}$ The overall sensitivity of the Captia Syph G was $98.3 \%$ : the test was $100 \%$ sensitive in treated syphilis and in all stages of untreated syphilis except primary infection (sensitivity $82 \%$ ). Because of its lower sensitivity in primary infection it was concluded that the Captia Syph G test did not seem to be a suitable replacement for the present screening combination of VDRL and TPHA tests but was an ideal confirmatory test. ${ }^{4}$ As this was not an "in-use" study there were no data to support the view that the VDRL and TPHA screening combination performs better than the Captia Syph G (with a sensitivity of $82 \%$ in primary syphilis) might in routine practice.

We reviewed the performance of the VDRL and TPHA screening combination over eight years of routine practice to provide a baseline for assessing screening by anti-treponemal IgG EIA.

\section{Methods}

We reviewed the notes and laboratory records for all patients with a diagnosis of untreated syphilis who attended the genitourinary medicine unit at Edinburgh Royal Infirmary between 1980 and 1990 inclusive. Between 1980 and 1987 all sera were screened by the combination of VDRL and TPHA tests. ${ }^{5}$ The FTA-ABS test was performed as an additional test whenever requested, as in the case of suspected early primary syphilis, or routinely whenever one of the screening tests was positive. A positive result in a screening test was confirmed by quantitative testing: the VDRL test was titrated to the end point whereas the TPHA titration was usually limited to a final serum dilution of 1 in 5120 . Titres were expressed as the reciprocal to a

Department of
Medical Microbiology,
University Medical
School, Edinburgh
EH8 9AG
H Young
A Moyes
Genitourinary
Medicine Unit,
Edinburgh Royal
Infirmary
A McMillan
J Patterson
Correspondence to:
Dr H Young
Accepted for publication
4 July 1991


Table 1 Diagnosis of syphilis 1980-1990 in patients attending genitourinary medicine unit in Edinburgh

\begin{tabular}{llclc}
\hline & $\begin{array}{l}\text { Primary } \\
\text { syphilis }\end{array}$ & $\begin{array}{l}\text { Secondary } \\
\text { syphilis }\end{array}$ & $\begin{array}{l}\text { Early } \\
\text { latent } \\
\text { syphilis }\end{array}$ & $\begin{array}{l}\text { All } \\
\text { other } \\
\text { stages }\end{array}$ \\
\hline 1980 & 7 & 7 & 6 & 26 \\
1981 & 7 & 8 & 6 & 21 \\
1982 & 5 & 9 & 5 & 14 \\
1983 & 11 & 11 & 5 & 17 \\
1984 & 10 & 6 & 10 & 17 \\
1985 & 1 & 5 & 6 & 9 \\
1986 & 3 & 0 & 0 & 7 \\
1987 & 0 & 1 & 1 & 8 \\
1988 & 0 & 2 & 1 & 12 \\
1989 & 0 & 1 & 0 & 4 \\
1990 & 4 & 2 & 4 & 4 \\
\hline
\end{tabular}

Primary $92 \%$ male; secondary $98 \%$ male; early latent $95 \%$ male; other stages $68 \%$ male.

final serum dilution yielding a positive reaction.

Whenever a TPHA titre of more than 5120 was found in association with a negative VDRL test on screening, a quantitative VDRL test was performed to exclude a false negative reaction due to the prozone phenomenon. Starting in 1988, all specimens were screened with EIA (Captia Syph G) as a single screening test. ${ }^{3}$ EIA plates were read at $450 \mathrm{~nm}$, on a Titertek Multiskan linked to our departmental DEC PDP 11/44 computer used for request input and report generation. Each test run was validated by running negative, low and high titre positive kit controls in duplicate. The validity of the run (mean absorbance of the negative control less than 0.25 ; mean absorbance of the low titre positive control more than 1.5 times that of the negative control and less than 0.5 times that of the high titre positive; and mean absorbance of high titre positive control more than $\mathbf{0 . 8}$ ) was checked by the computer before automatically converting the absorbance given by each test serum to an antibody index (absorbance of test serum/absorbance of low titre positive control). Specimens with an antibody index of less than 0.9 were considered negative and, unless additional tests had been requested, this result led to the issue of a computer generated negative report. Specimens with an antibody index of $\geqslant 0.9$ were retested and also examined by quantitative VDRL and TPHA tests, the FTA-ABS test, and by EIA for specific anti-treponemal IgM (Captia Syph M). ${ }^{3}$ Whenever requested, the FTA-ABS and Captia Syph $M$ tests were performed irrespective of the screening results.

Statistical analysis was performed using the $\chi^{2}$ test with Yates's correction.

Table 2 Pattern of serological tests results in 44 cases of untreated primary syphilis

\begin{tabular}{lllllll}
\hline & & & & & \multicolumn{2}{c}{ Dark ground microscopy } \\
\cline { 6 - 7 } VDRL & TPHA & FTA-ABS & $\begin{array}{c}\text { Number } \\
\text { of cases }\end{array}$ & Positive & Negative & None \\
\hline Negative & Negative & Negative & 5 & 5 & 0 & 0 \\
Positive & Negative & Negative & 3 & 2 & 0 & 1 \\
Negative & Positive & Negative & 1 & 1 & 0 & 0 \\
Negative & Negative & Positive & 2 & 1 & 0 & 1 \\
Positive & Positive & Negative & 0 & 0 & 0 & 0 \\
Positive & Negative & Positive & 3 & 2 & 0 & 1 \\
Negative & Positive & Positive & 4 & 0 & 3 & 1 \\
Positive & Positive & Positive & 26 & 14 & 5 & 7 \\
\hline
\end{tabular}

Equivocal reactions scored positive.
Results

Table 1 shows the pronounced decrease in the number of cases of syphilis diagnosed during the past decade.

During the period 1980-84 there were 208 cases of which $113(54.3 \%)$ were early infections; between 1985 and 1989 there were 61 cases of which $21(34.4 \%)$ were early infections: the decrease in the proportion of early syphilis is highly significant $\left(\chi^{2}=6.7 ; p<\right.$ 0.01 ). In 1990, however, there was a significant increase in the proportion of cases of early syphilis when compared with the period 1985$89\left(\chi^{2}=5 ; p<0.05\right)$. The pattern of serology and dark ground microscopy results for the 44 cases of primary syphilis diagnosed from 198087 is shown in table 2 .

A total of 11 patients did not have a dark ground investigation. The overall sensitivity of dark ground microscopy was $75 \cdot 8 \%$ (25 of 33) while $11.4 \%$ (five of 44 ) of cases were positive only by microscopy. Of the individual screening tests, the VDRL detected $72 \cdot 7 \%$ (32 of 44) and the TPHA $70.5 \%$ ( 31 of 44). The FTAABS was reactive in $79.5 \%$ (35 of 44 ) and was the most sensitive single test. The screening combination of VDRL and TPHA tests detected $84.1 \%$ (37 of 44 ) of cases. The VDRL and TPHA were complementary in detecting primary infection in that five cases were TPHA positive VDRL negative, while six cases were TPHA negative VDRL positive. The sensitivity of the VDRL and TPHA tests was similar in terms of reactivity above the cut-off point for screening, but the antibody titre was higher in the case of the VDRL test than in the TPHA (table 3).

Twenty six sera $(59 \%)$ gave a titre of $\geqslant 2$ in the VDRL test compared with 14 sera $(32 \%)$ giving an equivalent titre in the TPHA $\left(\chi^{2}=\right.$ 5.5; $\mathrm{p}<0.02)$. Four of the patients with primary infection had been treated for syphilis; three of these patients had a TPHA titre of $\geqslant 64$ units and a VDRL titre of 32.

Results of quantitative tests performed following screening are given for secondary syphilis in table 4 and for early latent syphilis in table 5.

One patient with early latent syphilis was omitted from the analysis because records were missing. All 85 cases of secondary and early latent infection were reactive in the FTA-ABS and TPHA tests. Quantitative VDRL testing

Table 3 VDRL and TPHA titres in 44 cases of untreated primary syphilis (1980-87)

\begin{tabular}{clc}
\hline & \multicolumn{2}{l}{ Number of cases with corresponding titre in: } \\
\cline { 2 - 3 } Titre & VDRL & TPHA \\
\hline 0 & 12 & 13 \\
Equivocal & 1 & 4 \\
1 & 5 & 13 \\
2 & 6 & 3 \\
4 & 8 & 1 \\
8 & 3 & 3 \\
16 & 2 & 1 \\
32 & 6 & 0 \\
$\geqslant 64$ & 1 & 6 \\
Total & 44 & 44 \\
\hline
\end{tabular}

TPHA titre given as units-that is, end point dilution/ screening dilution: $1=80 ; 2=160 ; \geqslant 64=\geqslant 5120$. 
Table 4 VDRL and TPHA titres in 47 cases of untreated secondary syphilis (1980-87)

\begin{tabular}{ccc}
\hline & \multicolumn{2}{c}{ Number of cases with corresponding titre in: } \\
\cline { 2 - 3 } Titre & VDRL & TPHA \\
\hline 0 & 1 & 0 \\
Equivocal & 0 & 0 \\
1 & 0 & 1 \\
2 & 3 & 1 \\
4 & 4 & 3 \\
8 & 7 & 6 \\
16 & 8 & 4 \\
32 & 10 & 8 \\
$\geqslant 64$ & 14 & 24 \\
Total & 47 & 47
\end{tabular}

Four sera $(8 \%)$ gave a prozone reaction in the VDRL test (titres of $16 ; 64 ; 128$ and 512 ).

Table 5 VDRL and TPHA titres in 38 cases of untreated early latent syphilis (1980-87)

\begin{tabular}{clc}
\hline & \multicolumn{2}{l}{ Number of cases with corresponding titre in: } \\
\cline { 2 - 3 } Titre & VDRL & TPHA \\
\hline 0 & 8 & 0 \\
Equivocal & 0 & 1 \\
1 & 3 & 1 \\
2 & 2 & 2 \\
4 & 4 & 8 \\
8 & 9 & 4 \\
16 & 3 & 1 \\
32 & 4 & 5 \\
64 & 5 & 16 \\
Total & 38 & 38
\end{tabular}

Four sera $(10 \%)$ gave a prozone reaction in the VDRL test (titres of 32; $128(2)$; and 512).

showed reactivity in $97.9 \%$ ( 43 of 44 ) of cases of secondary syphilis, but four of these sera $(8.5 \%)$ gave a false negative reaction on screening, presumably due to the prozone phenomenon. Therefore the sensitivity of the VDRL based on the initial screening results was $89.4 \%$ (42 of 47). Prozone reactions also occurred in early latent syphilis: the VDRL was reactive in $79 \%$ (30 of 38) of cases after repeat testing, but there were four false negative reactions initially, resulting in a screening sensitivity of $68 \%$ (26 of 38). Apart from the greater sensitivity of the TPHA in detecting early latent syphilis, titres were also significantly higher than in the VDRL test: titres of $\geqslant 16$ were found in $57.9 \%$ (22 of 38) TPHA results compared with $31.6 \%$ (12 of 38 ) VDRL tests $\left(\chi^{2}=4.3 ; p<0.05\right)$

Screening and quantitative results for the 14 cases of early syphilis diagnosed since the introduction of Captia Syph G as a single screening test are shown in table 6 .

Table 6 Serological results for 14 cases of early syphilis detected by screening with anti-treponemal IgG enzyme immunoassay

\begin{tabular}{lllrrl}
\hline Stage & $E I A-I g G$ & EIA-IgM & VDRL & TPHA & FTA-ABS \\
\hline Primary & 3.51 & $2 \cdot 12$ & 32 & 160 & Positive \\
Primary & 2.57 & 2.19 & 8 & 160 & Positive \\
Primary & 2.12 & 3.21 & 4 & 80 & Positive \\
Primary & 1.70 & 1.29 & 0 & 80 & Positive \\
Secondary & 2.57 & 1.37 & 16 & 2560 & Positive \\
Secondary & 1.31 & 3.22 & 32 & 2560 & Positive \\
Secondary & 3.12 & 2.07 & 128 & 5120 & Positive \\
Secondary & $4 \cdot 11$ & 1.33 & 128 & 5120 & Positive \\
Secondary & 4.54 & 2.95 & 64 & 5120 & Positive \\
Early latent & 3.35 & 1.51 & 4 & 2560 & Positive \\
Early latent & 3.72 & 1.15 & 16 & 5120 & Positive \\
Early latent & 1.49 & 2.96 & 2 & 640 & Positive \\
Early latent & 4.50 & 0.50 & 128 & 5120 & Positive \\
Early latent & 2.59 & 0.33 & 0 & 160 & Positive \\
\hline
\end{tabular}

IgG and IgM results expressed as an antibody index. Secondary with VDRL titre of 128 gave a prozone.
All four patients with primary infection gave positive tests for anti-treponemal IgG and were also reactive in the VDRL, TPHA, and FTAABS tests. Specific anti-treponemal IgM was found in $85.7 \%$ (12 of 14 ) of the patients: the two patients with early latent infection and negative IgM results had received antibiotics on at least one occasion in the two years preceding the diagnosis.

\section{Discussion}

In the United Kingdom the annual number of cases of syphilis declined between 1980 and $1986 .^{6}$ Recently, however, there has been a resurgence of heterosexually acquired syphilis in London ${ }^{7}$ while over the past year a similar phenomenon has been observed in Edinburgh (table 1). In Amsterdam the declining trend was reversed in 1987 when increases in heterosexually acquired infection were observed. ${ }^{8}$ In the United States the increase in heterosexually acquired infection observed in recent years has been associated with a very steep increase in congenital infection. ${ }^{9}$ These findings substantiate the case for antenatal screening, ${ }^{10-12}$ the value of which has been questioned in recent years. The potential for concomitant infection with the human immunodeficiency virus(HIV) to influence both the treponemal antibody response and the clinical course of syphilis ${ }^{1314}$ is an additional factor to consider in the selection and use of serological tests for syphilis.

The above changes in the epidemiology of syphilis necessitate a reappraisal of diagnostic methods. In spite of the recent increases, screening or case finding for syphilis involves very large numbers of specimens being examined to detect a very small number of positive findings. In these circumstances new methods such as EIA, with the potential for automated testing and computed reporting, have many attractions. Before replacing existing screening tests with a new protocol it is important to establish that the performance of the new method is comparable with that of the existing procedures.

The Captia Syph G gives high specificity and is sensitive in detecting anti-treponemal antibody in untreated as well as treated infection. ${ }^{34}$ Because the sensitivity in detecting untreated primary infection was $82 \%$ (compared with $100 \%$ for all other stages of infection), Lefèvre et $a l^{4}$ considered that this EIA was unsuitable as a replacement for the VDRL and TPHA screening combination. Our results show, however, that a sensitivity of $82 \%$ in detecting untreated primary infection is greater than any other single test, including dark ground microscopy (76\%), VDRL (73\%), TPHA $(71 \%)$ and FTA-ABS (80\%). Similar sensitivities for dark ground microscopy (78\%), VDRL (70\%), and TPHA $(75 \%)$ in untreated primary syphilis have also been reported. ${ }^{15}$ Although the combination of VDRL and TPHA tests did give slightly greater sensitivity $(84 \%)$, this value is not significantly different $(p>0.5)$ from the $82 \%$ sensitivity of EIA. ${ }^{4}$ The high sensitivity of EIA using anti-human IgG conjugate has been 
reported with both "in-house" and commercial EIA systems. Veldkamp and Visser ${ }^{16}$ detected all 32 cases of untreated primary infection, while the Bio-Enza Bead test was reactive in five of six cases ${ }^{17}$ and all three cases ${ }^{18}$ of untreated primary infection. In our study all four primary cases diagnosed since 1988 were reactive in the EIA for IgG. A double conjugate EIA ${ }^{19}$ for both IgG and IgM found that in infected patients a positive IgG result always accompanied a positive IgM result. It was also shown by western blotting that by the time clinical signs develop most patients have both IgG and IgM antibody. ${ }^{20}$

Routine serological screening will always fail to detect some cases of early primary infection. For example, in our study (table 2), $11 \%$ of cases were negative in all serological tests including the FTA-ABS. Anderson et $a l^{15}$ reported that $14.5 \%$ of 939 patients with primary syphilis were seronegative in all tests at the time of presentation. A high index of clinical suspicion is the key factor in making a diagnosis of primary infection and is probably more important than the serological screening schedule used.

Compared with the seronegative "window" in early primary syphilis, the occurrence of false negative results in secondary and early latent syphilis when very high antibody titres are found (tables 4 and 5 ) is a more serio-us and underestimated limitation of certain serological screening tests. The prozone phenomenon is generally considered to occur in $1-2 \%$ of patients with secondary syphilis. ${ }^{21}$ Spangler et $a l,{ }^{21}$ however, reported 24 patients in various stages of infection whose serological tests were negative; 16 of the 24 negative tests were due to the prozone phenomenon. Our findings (tables 4 and 5) that $8 \%$ of patients with secondary and $10 \%$ of patients with early latent infection gave a false negative result in the VDRL test due to the prozone phenomenon means that such tests should not be used alone to screen for untreated early infection.

The high rate of prozone reactions reported in our study may be related to our routine policy of performing a quantitative VDRL test of any serum giving a TPHA titre of $\geqslant 5120$. The tendency of the prozone phenomenon to occur in sera with high VDRL titres could be particularly important in patients with concomitant HIV infection as sera from many such patients have unusually high antibody titres. ${ }^{132}$ Unusually high titres are also found in patients undergoing reinfection, even in cases of primary syphilis (table 3 ). The increased risk of the prozone phenomenon occurring in sera from these patients makes detection of reinfection by non-treponemal tests less reliable. False negative VDRL reactions may also occur in the cerebrospinal fluid. Feraru et $a^{23}$ reported two HIV positive patients with neurosyphilis whose initial cerebrospinal fluid VDRL tests were negative. In one of these patients the initial negative VDRL test may have been due to the prozone phenomenon. ${ }^{23}$ False negative results are also important in antenatal screening. Recently four cases with false negative serological tests due to the prozone phenomenon were encountered in women who gave birth to infants with congenital infection. ${ }^{24}$ Although serum dilution before testing was recommended as a routine procedure for all seronegative women in an area of high syphilis prevalence, ${ }^{24}$ in our opinion this is not a practical proposition when all patients can be screened simply and reliably with specific antitreponemal antibody tests such as the TPHA or EIA.

Our results (table 6) indicate that detection of anti-treponemal IgG followed by testing for specific IgM antibodies by EIA is an efficient and simple screening method for detecting syphilis and indicating whether active disease is present. In our series all nine patients with primary or secondary syphilis were IgM positive compared with only $60 \%$ (three of five) of cases of early latent infection. Lefèvre also found that the correlation between detection of anti-treponemal IgM and active disease was high $90 \%$ (27 of 30 ) in primary and secondary infection but lower $64 \%$ (nine of 14 ) in early latent infection. ${ }^{4}$ The lower detection rate of specific IgM in early syphilis could be due to a combination of previous antibiotics for other conditions (as in the two negative cases in our series) and the general decrease in the spectrum and strength of antibody response which occurs with increased duration of infection. ${ }^{20}$

We conclude that in the case of primary syphilis there is no evidence to suggest that screening for anti-treponemal IgG by EIA is significantly less sensitive than the combination of VDRL and TPHA tests. Provided that clinicians are made aware of the "seronegative window" that may exist for one to two weeks during early primary infection, they maintain a high index of clinical suspicion, and have the facility to request additional tests, such as specific IgM or FTA-ABS, in cases of suspect primary infection, then there are many practical advantages and benefits in screening with a single test that lends itself readily to automation and computed report generation, thus overcoming problems of subjective interpretation and transcriptional errors.

1 Young H, Penn CW. Syphilis, yaws and pinta. In: Smith GR, Easmon CSF, eds. Topley and Wilson's principles of bacteriology, virology and immunology. Edward Arnold, 1990:588-604.

2 Luger AFH. Serological diagnosis of syphilis: current methods. In: Young H, McMillan A, eds. Immunological diagnosis of sexwally transmitted diseases. New York: diagnosis of sexwally transmitrec

3 Young H, Moyes A, McMillan A, Robertson DH. Screening for treponemal infection by a new enzyme immunoassay. Genitourin Med 1989;65:72-8.

4 Lefévre JC, Bertrand MA, Bauriaud R. Evaluation of the Captia enzyme immunoassays for detection of immunoglobulins $\mathbf{G}$ and $\boldsymbol{M}$ to Treponema pallidum in syphilis. J Clin Microbiol 1990;28:1704-7.

5 Young H, Henrichsen C, Robertson DHH. Treponema pallidum haemagglutination test as a screening procedure for the diagnosis of syphilis. Br J Vener Dis 1974;50: 341-6.

6 Public Health Laboratory Service Communicable Disease Surveillance Centre. Sexually transmitted disease in Britain: 1985-6. Genitourin Med 1989;65:117-21.

7 Tang A, Barlow D. Resurgence of heterosexually acquired early syphilis in London letter. Lancet 1989;ii:166-7.

8 van den Hoek. JA, van der Linden MM, Coutinho RA. Increase of infectious syphilis among heterosexuals in Amsterdam: Its relationship to

9 Progress toward achieving the 1990 objectives for the Nation for sexually transmitted diseases. Morbidity and Mortality Weekly Report 1990;39:53-7. 
10 Clay JC. Antenatal screening for syphilis. Br Med J 1989; 229:409-10.

11 Garland SM, Kelly VN. Is antenatal screening for syphilis worthwhile? Med J Aust 1989;151:368-70.

12 Gilbert GL. Congenital syphilis-should we worry? Med J Aust 1988;148:162-4.

13 Rufli T. Syphilis and HIV infection. Dermatologica 1989; 179:113-7.

14 Hicks CB, Benson PM, Lupton GP, Tramont EC. Seronegative secondary syphilis in a patient infected with the negative secondary syphilis in a patient infected with the
human immunodeficiency virus (HIV) with Kaposi human immunodeficiency virus (H1V

15 Anderson J, Mindel A, Tovey SJ, Williams P. Primary and secondary syphilis, 20 years' experience. 3: diagnosis, treatment, and follow up. Genitourin Med 1989;65: $239-43$.

16 Veldkamp J, Visser AM. Application of the enzyme-linked immunosorbent assay (ELISA) in the serodiagnosis of syphilis. Br J Vener Dis 1975;51:227-31.

17 Borobio MV, Alvarez-Dardet C, Gallardo RM. Evaluation of a solid-phase enzyme immunoassay in the diagnosis of of a solid-phase enzyme immunoassay in the

18 Stevens RW, Schmitt ME. Evaluation of an enzyme-linked immunosorbent assay for treponemal antibody. J Clin Microbiol 1985;21:399-402.

19 Farshy CE, Hunter EF, Larsen SA, Cerny EH. Doubleconjugate enzyme-linked immunosorbent assay for immunoglobulins $\mathbf{G}$ and $M$ against Treponema pallidum. J Clin Microbiol 1984;20:1109-13.

20 Baker-Zander SA, Hook EW, Bonin P, Handsfield HH, Lukehart SA. Antigens of Treponema pallidum recognized by IgG and IgM antibodies during syphilis in hized by IgG and IgM antibodies $J$ Infect Dis 1985;151:264-72.

21 Spangler AS, Jackson JH, Fiumara NJ, Warthin TA. Syphilis with a negative blood test reaction. JAMA 1964; 89:87-90.

22 Morbidity and Mortality Report. Centers for Disease Control, Atlanta. Recommendations for diagnosing and treating syphilis in HIV-infected patients. Arch Dermatol 1989;125:15-6.

23 Feraru ER, Aronow HA, Lipton RB. Neurosyphilis in AIDS patients: initial CSF VDRL may be negative. Neurology 1990;40:541-3.

24 Berkowitz K, Baxi L, Fox HE. False-negative syphilis screening: the prozone phenomenon, non-immune Obstet Gynecol 1990;163:975-7. 